Sección uno: Ensayo

Mujer y Educación. Un largo camino hacia la igualdad

\title{
Logros y desafíos de la (co)educación: un camino violeta por recorrer $^{1}$
}

Achievements and challenges of (co)education: a violet road ahead

\author{
Begoña Sánchez Torrejón \\ Universidad de Cádiz \\ begonia.sanchez@uca.es \\ Ana Álvarez Balbuena \\ Universidad de Cádiz \\ anaalvbal1@gmail.com \\ María Escribano Verde \\ Universidad de Cádiz \\ maria.escribanoverde@gmail.es
}

\section{Resumen}

A lo largo del artículo planteamos un análisis del recorrido histórico de la coeducación hasta llegar a la actualidad, seguidamente esbozaremos las diversas conquistas conseguidas y también los múltiples desafíos que todavía los centros educativos deben traspasar para lograr una escuela igualitaria entre niñas y niños. A pesar del largo camino recorrido, de las numerosas leyes decretadas en favor de la igualdad y del impulso del feminismo de los últimos años, la coeducación sigue siendo una asignatura pendiente para la escuela. Partiendo del objetivo número 5 sobre "Igualdad de género" marcado por los Objetivos de Desarrollo Sostenible (ODS) propuesto por la Organización de las Naciones Unidas (ONU), consideramos clave para la consecución de este objetivo, la coeducación como camino hacia una escuela y una sociedad igualitaria entre niñas y niños.

\footnotetext{
${ }^{1}$ Recibido:28/01/2021 Evaluado:15/02/2021 Aceptado: 02/03/2021
} 
Palabras clave: Coeducación, Género, Igualdad, Educación Inclusiva, Objetivos de Desarrollo Sostenible

\begin{abstract}
Throughout the article we raise an analysis of the historical tour of coeducation up to the present day, then we will outline the various achievements and the multiple challenges that schools still must overcome to accomplish an egalitarian school for girls and boys. Despite the long road travelled, the numerous laws decreed in favor of equality and the momentum of feminism in recent years, coeducation continues to be the unfinished business for schools. Based on Goal 5 about "Gender Equality" set by the Sustainable Development Goals (SDGs) proposed by the United Nations (UN), we consider coeducation as a key to achieve this goal, as a path towards an egalitarian school and society for girls and boys.
\end{abstract}

Keywords: Coeducation, Gender, Equality, Inclusive Education, Sustainable Development Goals

\title{
Introducción
}

Teniendo en cuenta los desafíos a superar marcados por los Objetivos de Desarrollo Sostenible (ODS) propuestos por la Organización de las Naciones Unidas (ONU), la igualdad de género constituye uno de los principios de la Agenda 2030, particularmente, el objetivo número 5 sobre "Igualdad de género". La Agenda 2030 para el Desarrollo Sostenible impulsa el compromiso de toda la comunidad internacional para el logro de la igualdad de género y el empoderamiento de todas las mujeres y de las niñas a través de un objetivo específico y de forma transversal en otros objetivos. Otro de los objetivos relevante es el objetivo número 4 "Educación de calidad", el reto a superar es conseguir una educación de calidad inclusiva y equitativa, con la que se debe promover oportunidades de aprendizaje permanente para todos. Para que la agenda 2030 avance en su ejecución, consideramos necesaria la coeducación como camino hacia una escuela y una sociedad igualitaria entre niñas y niños, libre de violencias machistas. Ballarín (2001), señala que la coeducación se basa en el principio de igualdad entre hombres y mujeres, por ello partimos de un marco legislativo sobre igualdad, que debemos plasmar en medidas concretas en nuestros centros educativos. Debemos tener en cuenta que los centros escolares tienen una gran responsabilidad como medio de transmisión de valores no sexistas para los niños y las niñas, que en un futuro serán ciudadanos y ciudadanas en igualdad. Subirats (1994) comenta que la escuela "no puede eliminar por sí solo las diferencias insertas en el conjunto de la sociedad, pero el cambio ha de producirse en algún punto o momento, o en varios... y la educación es una pieza esencial para el cambio" (p.73).

En este sentido, la escuela coeducativa es una herramienta clave para consolidar experiencias democráticas que favorezcan, la igualdad de género y una escuela inclusiva. Así mismo, para poder lograr una sociedad más justa y equitativa, es prioritario desarrollar políticas educativas 
que priorizan la coeducación, capaces de suprimir los estereotipos sexistas que acrecientan la desigualdad de género. La meta principal de la coeducación es educar en igualdad de género, superando las desigualdades existentes, promoviendo modelos educativos igualitarios (Gallardo-López y Gallardo Vázquez, 2018). Uno de los retos que debe superar el sistema educativo es el desafío de la igualdad como una necesidad social imperante para poder avanzar en igualdad y democracia (Pérez Carracedo, 2006). Colás Bravo (2004), postula que es necesario para seguir avanzando en la coeducación, plantear teorías innovadoras, formas de actuación y modelos reales para poder incluir la cultura de la igualdad de género en los currículums educativos. A continuación, comenzaremos mostrando el recorrido histórico de la coeducación hasta llegar a nuestros días, para plantear los diversos avances conseguidos y también los desafíos que todavía debemos superar para lograr una escuela igualitaria entre niñas y niños. Finalizaremos con las conclusiones que abren diversas reflexiones para seguir avanzando en coeducación y poder alcanzar las metas propuestas en materia de igualdad y educación por los Objetivos de Desarrollo Sostenible (ODS).

\section{Recorrido histórico de la coeducación}

La coeducación es un concepto utilizado para simbolizar de manera precisa la educación de los niños y las niñas, en palabras de Subirats: "hace referencia a la educación conjunta de dos colectivos humanos específicos: los hombres y las mujeres" (1994, p.49). En el recorrido histórico de la coeducación en España, es necesario aludir a las limitaciones que ha ido sufriendo durante los años, antagónicamente, es necesario mencionar los avances que se han generado, gracias a los cambios sociales hacia la mujer, en otras palabras, debido a la aspiración en sus propósitos por desear la igualdad entre hombres y mujeres (GuerreroPuerta, 2017). Partiendo de la Ley de Instrucción Pública de 1857, popularmente conocida como la "Ley Moyano", defendió secularización de la enseñanza, es decir, la educación dejo de ser competencia de la iglesia para ser gestionada por el Estado (Quintana, 1975). Aun siendo visible la separación de la iglesia con la educación, se observa la desigualdad de género con aulas separadas por sexos e incluso una llamativa brecha salarial entre los maestros y las maestras, siendo las mujeres las grandes perjudicadas en este aspecto. Asimismo, en las materias ofrecidas a los niños existen cierta distinción a las que estudiaban las niñas, por ejemplo, las chicas dejaban de lado la geometría o las nociones de física, para centrarse en labores e higiene doméstica (Montero, 2009).

Ahora bien, se contemplaron progresos en la educación femenina de la época, ya que se reconoció, con carácter obligatorio, la educación primaria para mujeres con la creación de escuelas para niñas en pueblos pequeños y la formación pedagógica de las docentes fue notable con las Escuelas Normales femeninas. Sin embargo, estas nuevas escuelas de formación para las docentes solo hacían hincapié en las asignaturas relacionadas con las labores del hogar, consiguiendo así que una maestra tuviera escasos conocimientos sobre materias instrumentales (González, 2010). La ley Moyano aportó al mismo tiempo una sucesión de avances en materia, como son: una regulación en los niveles educativos, un control de asistencia del alumnado, pruebas específicas para pasar de un nivel a otro, así como una formación definida para obtener los títulos de maestro y maestra. No obstante, la educación de la mujer seguía siendo inferior a la del hombre, ya que solo la formaban para el trabajo en el hogar o el de magisterio (Geraldine, 1987). 
Posteriormente, en 1876 se desarrolló el proyecto educativo de la Institución Libre de Enseñanza (ILE), defendida por Francisco Giner de los Ríos (Pericacho, 2015). La ILE recogió el término coeducación en sus principios pedagógicos. Luchó por que la educación femenina alcanzará la misma importancia que la educación masculina, es decir la mujer debía educarse con el hombre y no como el hombre (Losada, 2019).

En lo concerniente a la formación concreta que debía desarrollar el profesorado de la época, recogía como requisito principal una buena conducta religiosa y moral. Con referencia a la formación inicial del profesorado, Sánchez y Barea (2019), nos hablan del papel preponderante que tiene el profesorado para la construcción de una cultura de género y así conseguir una sociedad igualitaria entre hombres y mujeres. En el mismo orden de ideas, es imprescindible ofrecer una formación a los docentes y las docentes para la eliminación de las discriminaciones y estereotipos sexistas en el sistema educativo. En tal sentido, se destaca la falta de formación e intervención de las mujeres en altos cargos en esta etapa histórica (Geraldine, 1987). Por lo que en la formación femenina se le daba prioridad a otras temáticas que tenían una visión más retrograda en relación con la igualdad de ambos sexos.

Uno de los componentes más importantes sobre el desarrollo histórico de la coeducación es la proclamación de la Segunda República en España en 1931. La idea que se quería conseguir era la de crear una educación igual para todos y todas, el laicismo y establecer una escuela sin discriminación por sexo, riqueza, nacimiento, ideología o religión, es decir, el camino hacia la coeducación e inclusión (Alcón, 2005). Resumiendo lo planteado, la religión quedó apartada del ámbito educativo, dando prioridad en las escuelas al conocimiento del alumnado y construyendo un acceso libre a la enseñanza para todos y todas sin distinción alguna (Pérez, 2000). En consecuencia, el número de mujeres que comenzaron a incorporarse en las escuelas superiores como docentes fue en aumento, ya que anteriormente las mujeres podían obtener el título de licenciatura, pero no ejercer en la práctica (Grana y Martín, 2015).

Es preciso tener en cuenta la etapa educativa existente durante los años de dictadura en España, donde se hacía latente un retroceso en aquellas pinceladas de evolución sobre coeducación destacadas anteriormente. Una de las esencias de identidad de este régimen político era el poder que le otorgaba a la iglesia y a la política dentro de las instituciones educativas. Tal es el caso, como alude Fuertes (2015), hubo una modificación significativa en el plan de estudios, que restableció las materias de Religión e Historia Sagrada con carácter obligatorio. El objetivo primordial de las escuelas era que el alumnado dejará de lado la formación, dando el papel principal en su vida a la religión y el patriotismo. A causa de estas modificaciones se puede concretar dicha regresión en materia de coeducación, es decir, la sociedad defendía el papel del hombre como aquel que debía realizar los trabajos fuera de casa y mantener a su familia, y por otro lado el rol de la mujer estaba centrado en las labores doméstica y crianza de los hijos e hijas.

No podemos dejar de lado un aspecto fundamental en materia educativa como es la formación del profesorado, que durante este periodo también sufrió reestructuraciones. Por ejemplo, se perseguía contar con un profesorado afín al régimen y por ello se sustituyeron muchos docentes que defendían a la República. Además, las instituciones de enseñanza del profesorado también contaron con numerosas transformaciones. Sostenían un control exhaustivo del adiestramiento político hacia los docentes, dominado en las Escuelas Normales de Magisterio y los centros privados de la iglesia. Al mismo tiempo, se ejercía un 
control en su trabajo diario en las aulas y sus ideales políticos (Fuertes, 2015). En relación con este tema, la incidencia católica en el sistema educativo en el periodo franquista realza la idea de la separación de sexos en los centros, y además crece la diferenciación por clases sociales.

Paralelamente se desarrollaba un camino diferente para las maestras en el régimen, Grana y Martín afirman que fue: "una regresión con respecto a las incipientes políticas republicanas en favor de la incorporación de la mujer a la sociedad como sujeto de pleno derecho" (2015, p. 61). Como consecuencia de estas nuevas medidas, las mujeres solo podían acceder a aquellos puestos de trabajos que el régimen permitía. Se crearon Escuelas del Hogar de los Institutos Nacionales Femeninos de Enseñanza Media, en las que trabajaban maestras centradas en materias relacionadas con el campo doméstico (Grana y Martín, 2015). En los últimos años de la dictadura de Francisco Franco, el ministro de Educación José Luis Villar Palasí impulsó la Ley General de Educación (LGE) de 1970. Donde se recogían ideas disconformes para el Régimen. En efecto, la LGE, demandaba una educación general básica igual para todos y todas. Además, en relación con la educación universitaria, aunque estaban aferrados a los valores franquistas de diferenciación de clases y el protagonismo de la iglesia, exigía una educación diferente adaptada a la sociedad (Gómez, 2000).

\section{La coeducación en la actualidad}

En 1977 se produce en España un cambio histórico, se desarrollaron las primeras elecciones generales desde los tiempos de la Segunda República y tras finalizar la dictadura de Francisco Franco. Las cortes que salieron elegidas aprobaron la Constitución de 1978 y con ella se da comienzo al periodo democrático español. La década de los ochenta marcó un antes y un después en la educación, ya que se apuesta por movimientos de transformación pedagógica, es decir, una educación integral, liberadora, critica, fomento de la coeducación, enseñanza no confesional, formación continua del profesorado, carácter obligatorio en secundaria, tolerancia, solidaridad etc.

Con el inicio de los noventa llegó la democratización de la educación, consecuencia de la transformación social que surgió en el país en los últimos años y repercutiendo en una notoria eficacia en la calidad del sistema educativo (Gómez, 2000). Se aprobó la Ley General del Sistema Educativo (LOGSE) de 1990, que defendía la coeducación como eje significativo en su narración. Además, ponía de manifiesto la obligación y necesidad de suprimir las diferencias de género que generaba la propia labor educativa. En otras palabras, se requería trabajar para que las mujeres pudieran acceder de igual forma a las carreras científicas o técnicas, además de al mercado laboral de un determinado campo profesional del cual habían sido apartadas. El análisis procedente centra su atención en la Ley Orgánica de Educación (LOE) en 2006, los puntos más controvertidos de esta ley fueron el cambio de la asignatura de religión de obligatoria a voluntaria, aunque todos los centros debían ofrecerla. Por otro lado, incluyeron la materia de Educación para la Ciudadanía y los Derechos Humanos. Asimismo, podemos ver que en su narración contempla la coeducación y el impulso hacia la igualdad efectiva entre hombres y mujeres, con el objetivo de favorecer la homogeneidad de derechos y oportunidades verdadero entre ambos sexos (Ley Orgánica de Educación, 2006).

Dentro de este marco legislativo en educación abordamos la Ley Orgánica para la Mejora de la Calidad Educativa (LOMCE), aprobada en 2013. Se produjo un retroceso en la calidad de 
la coeducación, ofreciendo ayudas públicas a colegios que fomentaban la segregación por sexos en las aulas, además de volver a ofrecer el carácter obligatorio a la asignatura de religión. Se recogen ciertas modificaciones en la LOMCE, que expresaba: "el desarrollo, en la escuela, de valores que fomentan la igualdad efectiva entre hombres y mujeres, así como la prevención de la violencia de género." (2013, p.11). También recoge la igualdad de oportunidades entre ambos sexos para acceder a cualquier tipo de profesión. (Ley Orgánica para la mejora de la calidad educativa, 2013).

Por último, contamos con la Ley Orgánica de modificación de la Ley Orgánica de Educación (LOMLOE) de 2020, siendo la legislación educativa más actual. Otorga un enfoque significativo de igualdad de género, desenvolviendo en todas las etapas de aprendizaje la coeducación, la prevención de la violencia de género, además hace referencia al respeto de la diversidad afectivo-sexual y familiar, creando un camino inclusivo y no sexista (Instituto Asturiano de la Mujer, s.f.). Hay que destacar en la etapa de educación primaria, incorporan al curriculum una nueva asignatura denominada Educación en Valores Cívico y Éticos, con el fin de trabajar aspectos relacionados con los Derechos Humanos y de la Infancia, a la importancia del desarrollo sostenible, además de la igualdad de mujeres y hombres, teniendo presente el respeto a la diversidad. Por otro lado, en educación secundaria focalizan el objetivo en la incorporación de la perspectiva de género y respetar la diferencia de sexos. Sin olvidar, eliminar los estereotipos que generen discriminación entre ambos sexos, tan significativos en este periodo educativo debido a la sensibilidad de los educandos (Instituto Asturiano de la Mujer, s.f.).

Dentro de este orden de ideas, defiende y da prioridad a los centros educativos que trabajen con grupos heterogéneos en las aulas sin discriminación de género o por su orientación sexual. Otro aspecto importante, es la renovación del material utilizado dando el lugar que le corresponde a las mujeres tanto a nivel histórico como actual, es decir, favorecerá un currículo que no contengan estereotipos sexistas o discriminatorios. (Proyecto de Ley Orgánica por la que se modifica la Ley Orgánica de Educación, 2020). Otro punto para destacar en la narración de esta ley es la utilización tan necesaria de un lenguaje inclusivo que represente a todas y todos los miembros de la sociedad. Es necesario respetar el poder de las palabras para realizar las modificaciones necesarias, para conseguir el respeto y la tolerancia hacia los demás y las demás dejando de lado las diferencias. Con todo lo mencionado anteriormente, se persigue un cambio en las acciones sociales de sensibilización hacía la violencia de género. En otras palabras, se quiere visibilizar la damnificación que produce la discriminación y los actos violentos hacia las mujeres. Asimismo, llevar las actuaciones de sensibilización a los niños y las niñas y adolescentes consiguiendo que construyan el valor de igualdad para la mejora de la convivencia entre hombres y mujeres (Instituto Asturiano de la Mujer, s.f.).

Para finalizar con el análisis de la LOMLOE y sus modificaciones, se debe mencionar los Objetivos de Desarrollo Sostenible (ODS), propuestos por la Organización de las Naciones Unidas (ONU). Para hacer cumplir todas las modificaciones recogidas en esta ley cuentan con un periodo de diez años para lograr su implantación. Se defiende ofrecer y asegurar una educación inclusiva equitativa y de calidad, además impulsar oportunidades de aprendizaje a lo largo toda la vida para todos y todas. Persiguen eliminar las diferencias de género en la educación y garantizar la accesibilidad igualitaria a todos los niveles de la enseñanza y formación profesional (Organización de las Naciones Unidas, s. f.). Hay que destacar, entre 
los diecisiete ODS, que observamos dos que tienen mayor conexión con el tema que nos concierne. En primer lugar, el objetivo número 4, que defiende una educación de calidad, inclusiva y equitativa, garantizando la igualdad de oportunidades para todos y para todas. En segundo lugar, se habla del objetivo número 5, que se centrar en trabajar la igualdad de género, centrándose en la labor principal de empoderamiento de mujeres y niñas (Organización de las Naciones Unidas, s. f.).

Las normativas de educación deben tener presente la evolución en la sociedad española, en la formación que reciben sus jóvenes y en la necesidad de lograr un bienestar colectivo e individual. La actuación educativa es la herramienta principal para la construcción de nuestro alumnado, y como resultado la de los futuros ciudadanos y las futuras ciudadanas de nuestro país. En consecuencia, la educación es el medio para renovar o modificar la cultura, los conocimientos y los valores que alimentan a la sociedad, por ello es necesario trabajar para desarrollar una convivencia democrática y el respeto hacia la diversidad en todos sus campos (Ballarín, 2017). Se debe tener presente que la educación es un campo que debe ir ajustándose según la evolución que va desarrollando la sociedad, es decir, tiene un carácter dinámico con la necesidad de actualizarse continuamente. Este carácter renovador es el que persigue crear sistemas educativos competentes que ayuden a sustentar una sociedad basada en la igualdad.

\section{Logros de la coeducación}

Haciendo balance de los logros alcanzados en niveles de igualdad desde las prácticas coeducativas, que vienen determinados directamente por el propio avance sociocultural en materia de educación, encontramos variabilidad de pasos importantes alcanzados desde la coeducación española. De tal forma, partimos del hecho de que el s. XX ha sido definido como el siglo de las mujeres, haciendo referencia al avance en materia de coeducación (del Amo, 2009), aunque es necesario resaltar que la evolución hacia la equidad no ha sido de manera totalmente progresiva, sino que se aprecian avances, estancamientos y retrocesos.

A nivel general, los logros en materia de coeducación que se van a comentar en este apartado, parten, por un lado, de la igualdad de oportunidades para acceder al sistema educativo desde la perspectiva de equidad de género y, por otro lado, de la posibilidad del género femenino de participar en la gestión del sistema educativo y en las experiencias laborales de dicho sector, es decir, partimos desde la etapa histórica en la cual se comienza a experimentar la aceptación social de que el género femenino obtuviese derecho al conocimiento y, además, la implicación del género masculino en esa participación femenina (Aristizabal, 2011). Dicho de otro modo, los logros que se van a tratar parten del desarrollo legislativo, político y social alcanzado por la sociedad moderna y su configuración como factor en los logros coeducativos. Dado que se propicia la elaboración de leyes más justas e igualitarias para todos los sectores que la integran, permitiéndose una mejora global en la situación del género femenino, concretamente en su participación en las actividades educativas y su incorporación al mundo laboral (Arjona, Díaz y Rico, 1999).

Teniendo en cuenta esta realidad, uno de los logros más importantes alcanzados se centra en la formación del profesorado en materia coeducativa. Así, dicha formación se presenta como una preocupación central a la hora de lograr la promoción de la igualdad entre géneros en educación, sobre todo, porque esta debe de centrarse en potenciar el desarrollo de modelos y experiencias formativas que potencien intervenciones pedagógicas de carácter coeducativo. 
Sin embargo, aún nos encontramos en los primeros pasos de la ardua tarea de formación del profesorado en coeducación y esto, entre otras realidades, provoca que la situación de implementación de formación específica al profesorado de las etapas de Educación Infantil y Primaria, en términos de coeducación, es heterogénea en el caso del conjunto de Comunidades Españolas (Fuentes-Guerra, Luque y Freixas, 2007; Jiménez, Vega y RebolloCatalán, 2013). Dicha formación viene vinculada, además, con transformaciones en las condiciones curriculares. De tal modo, se hace necesario remarcar la figura de los materiales didácticos y como se ha ido realizando trabajos de investigación relacionados con la perspectiva sexista y androcentrista de dichos materiales y su influencia en la práctica. De hecho, se puede visualizar guías de buenas prácticas para la elaboración de materiales con perspectiva coeducativa y guías para detectar el grado de sexismo y los estereotipos de género implícitos en cualquier material didáctico (Vaillo, 2013). En este punto, también se debe resaltar el papel activo de las administraciones a la hora de desarrollarse dichas investigaciones y, junto a ellas, programas suscitados al respecto como, por ejemplo, la guía específica, elaborada por la Junta de Andalucía, incluida como medida en el II plan estratégico de igualdad de género en educación 2016-2021, para el reconocimiento de estereotipos y sesgos sexistas en los materiales didácticos y curriculares (Consejería de Educación, 2016).

Otro hecho remarcado, vinculado con el anterior, es la contribución de las universidades en materia de contenidos educativos e investigación. Por ejemplo, a través del planteamiento de investigaciones relacionadas con temáticas relativas a las mujeres, tanto a nivel de crítica de los paradigmas clásicos, como de recuperación de la memoria perdida y de introducción de nuevas problemáticas y paradigmas de estudios. Resaltando, además, el hecho de que estas investigaciones han estado contando, en gran medida, con financiación pública desde organismos autonómicos diversos como es el caso del Instituto de la Mujer (Subirats, 2014). Con todo ello, la realidad coeducativa se potencia en la actualidad a través la puesta en marcha de medidas educativas dirigidas a la consecución de la igualdad de carácter europeo, nacional, autonómico y local (García-Pérez et al., 2014). Asimismo, se introduce en la agenda política institucional de nivel superior (Ullastres, 2014). Un ejemplo que remarcar en dicha agenda se visualiza en la relación de los diecisiete ODS de la agenda 2030, entre los cuales se observan dos objetivos con alto grado de conexión con la temática del escrito y que potencian acciones relacionadas, en cierta medida, con los estándares de la coeducación. En una primera instancia, el objetivo número cuatro en el cual se persigue la garantía de una educación inclusiva, equitativa y de calidad y, en una segunda instancia, en el objetivo número cinco en el cual se pretende el logro de la igualdad entre géneros y el empoderamiento de mujeres y niñas (Organización de las Naciones Unidas, s. f.).

Tal y como hemos visualizado, se establecen grandes avances en el recorrido de la coeducación e integración del concepto en la escuela, además de percibirse un aumento, de forma significativa y contundente, del número de programas, guías y talleres, junto a publicaciones, actividades de divulgación e investigaciones que tornan sobre las prácticas coeducativas, prestándose, sobre todo, atención a la transmisión cultural de estereotipos de género que se estable en la base del currículo oculto y en la jerarquía del conocimiento (Cueto, López y García, 2016; Duque, Fisas y Valls, 2004), sin embargo, tal como señala Subirats (2009), aún quedan retos y desafíos diversos para continuar creciendo, así, "hoy por hoy en la escuela, perduran elementos que reproducen y perpetúan el sexismo y las 
diferencias entre los futuros hombres y mujeres que formaran nuestra sociedad" (Instituto de la Mujer, 2008, p. 11).

\section{Retos futuros de la coeducación}

Ante los logros que se han ido alcanzando sobre la igualdad, como hemos podido reflejar, se percibe el hecho que de que es claramente visible la no consecución de una igualdad efectiva en la sociedad actual. Por ello, es necesario continuar avanzando a través de la transformación en todos los ámbitos socioeducativos. El punto principal para cambiar la realidad discriminatoria y desigualitaria en materia de género es poner en práctica una educación igualitaria que rompa con los fundamentos patriarcales que sustenta nuestra sociedad, y en la actualidad, se nos presenta como reto para ello, la propia legislación educativa vigente. Pese al largo camino histórico y la variabilidad de acciones del feminismo de los últimos años y el decreto de numerosas leyes en favor de la igualdad, la coeducación sigue siendo una realidad pendiente en el sistema educativo. Una cuestión que se apoya en los ODS de la agenda 2030 donde se marcan dos objetivos, por un lado, el objetivo número cuatro sobre "Educación de calidad" y, por otro lado, el número cinco sobre "Igualdad de Género", desde los cuales es posible considerar la coeducación como una de las prácticas clave para la consecución de dichos objetivos.

Desde esta perspectiva, a lo hora de percibir los nuevos retos y desafíos de la coeducación, es necesario prestar atención al hecho de que la desigualdad en las instituciones educativas no ha desaparecido, sino que se ha transformado a realidades más sutiles. Así, en el análisis de los centros educativos de manera global desde la perspectiva de género, se pueden observar una serie de prácticas que muestran evidencias claras de desigualdades por razón de género, siendo cada una de estos retos y desafíos aún por alcanzar desde la coeducación. Estas evidencias que son posible de apreciar se enmarcan, por ejemplo, en el lenguaje utilizado (Vargas, 2012), la ocupación de los espacios escolares (Romero, 2015) y las pautas en las relaciones entre géneros (Gallardo-López y Gallardo-Vázquez, 2018). Teniendo en cuenta estas evidencias, un desafío básico de la coeducación es desenmascarar los estereotipos de género, junto a la adaptación de metodologías educativas que desarrollen de manera integral a las personas, partiendo de las diferencias, pero evitando que estas se conviertan en motivo de jerarquización (Pérez y Gargallo, 2008).

Otro de los pilares sobre los que se basa los retos futuros de la coeducación es el profesorado. Aunque uno de los logros alcanzados en la coeducación son los programas de formación del profesorado, se pone de manifiesto, en diversas investigaciones, la resistencia en las actitudes de los profesionales de la educación hacia la igualdad y la coeducación, experimentándose bajo nivel de competencias relacionales y prácticas necesarias. Por ello, aún queda una transformación importante que recae en el replanteamiento de los planes de estudio de las distintas universidades en referencia a la coeducación e igualdad, sobre todo, en aquellos vinculados a estudios para profesionalización de educadores y educadoras, además, de seguir planteándose formación permanente para dichos profesionales en temática de igualdad (Bejarano, Mateos, 2014). Continuando con la figura del profesorado, se contempla como reto a alcanzar que estos, además, trabajen de forma colaborativa dado que, siguiendo las aportaciones de Baena y Ruiz (2009), “difícilmente se podría transmitir al alumnado una visión integradora, tolerante, democrática e igualitaria de la educación si en la misma 
organización, que dice promover estos valores, se observan conductas competitivas, excluyentes y segregacionistas" (p. 120).

Otra barrera a superar, es la comunidad educativa que, tal como señalan Uria, Leone y Morales (2019), debe de visualizarse como núcleo clave para la consecución de escuelas coeducadoras a través de su activa participación. Junto a ello, también recae como reto la formación de la comunidad educativa y facilitar recursos diversos para el trabajo, tanto individual como colectivo, de temas como las relaciones de poder, discriminaciones y violencias o interseccionalidad de las opresiones, es decir, género, sexualidad, lenguaje, capacitacismo, etcétera. Para progresar, por tanto, en la creación de escuelas coeducativas, Subirats (2010), plantea la necesidad como pilar principal para el avance real, el reconsiderar toda la cultura escolar a la luz de valores tradicionales femeninos, mostrando la importancia humana de estos y dándoles mayor relevancia en el mundo educativo. Junto a esta realidad, se podría considerar como factor para la evolución en los planteamientos coeducativos el establecer planes de trabajo para modificar las pautas sexistas y crear mecanismos de intervención para el cambio, el analizar los elementos de poder y autoridad presentes en los centros educativos y gestionar hasta qué punto ocultan o merman la presencia de mujeres e invisibilizan a las niñas y, además, modificar la distribución de poder en los centros educativos de manera que los cargos de toma de decisiones tiendan a ser más paritarios. Por último, ante el hecho de que el enfoque coeducativo actual no da respuesta a la igualdad que se persigue, un reto importante para el sistema educativo es que debe de sobrepasar este modelo de igualdad de oportunidad que persigue la coeducación y transitar hacia una nueva perspectiva, la escuela queer, la cual se torna como realidad relevante y necesaria al aportar herramientas útiles en el repensar educativo, más allá de la normalización binarista (Sánchez, 2017). Por todo ello, es posible concluir como desde diversas investigaciones se plantean multitud de retos para la coeducación en la actualidad. De tal forma, es relevante considerar que la igualdad real sigue siendo un desafío para los sistemas educativos, aunque exista un camino extenso recorrido y variabilidad de legislación en favor de la misma (Calatrava, 2013).

\section{Conclusiones}

Como hemos reflejado, todavía son varios los hándicaps que la escuela coeducativa debe traspasar, pero la construcción de una escuela no sexista, no permite más aplazamientos en nuestro sistema educativo, ya que desde ese contexto podemos conseguir los retos marcados por los Objetivos de Desarrollo Sostenible (ODS) e influir de manera equitativa en las vidas y las visiones de las nuevas generaciones en la construcción de una sociedad igualitaria. Camas (2015), defiende que "en las sociedades democráticas contemporáneas la igualdad constituye un principio fundamental para la convivencia. Un principio que vincula a todas las personas en dignidad, respeto y derechos independientemente de su condición o circunstancia" (p. 75). La igualdad de oportunidades entre mujeres y hombres debe ser un objetivo común de toda sociedad democrática y para conseguirlo el sistema educativo debe priorizar la coeducación y aunar esfuerzos para conseguir la toma de conciencia respecto a la eliminación de roles y estereotipos de género.

A modo de conclusión, el profesorado es uno de los protagonistas en la creación de una escuela coeducativa, es una figura determinante en el cambio de la desigualdad en las aulas 
entre el alumnado, por ello consideramos que su formación con perspectiva de género tanto inicial como continua, es un camino hacia la consecución de los Objetivos de Desarrollo Sostenible (ODS) con respecto a la educación y a la igualdad. Además a través de la reflexión de su práctica docente, este debe asumir un papel protagonista en la revisión de sus propios conceptos y prácticas docentes con el fin de ofrecer nuevas experiencias igualitarias al alumnado, libres de sexismo (Sánchez y Soto, 2020). Somos conscientes del papel de la coeducación como herramienta necesaria en la prevención de la violencia de género y, por lo tanto valoramos que debe estar presente en la formación del profesorado como elemento inclusivo en la igualdad de oportunidades entre niños y niñas. Tomé (2017), plantea que para conseguir una escuela coeducativa, necesitamos propuestas globales que impliquen a toda la comunidad educativa, para ello, es imprescindible involucrar a todos los miembros que componen la familia educativa. Por todo esto, es necesario para que exista una continuidad del trabajo coeducativo del centro en las familias, que estas tengan una visión libre de estereotipos sexistas, para ello, consideramos preciso trabajar con las familias de manera conjunta. Otro aspecto a tener en cuenta para lograr una educación inclusiva, equitativa y de calidad como nos plantea la Agenda 2030, es el empoderamiento de las niñas en el ámbito tecnológico para superar la brecha digital (Pérez González y Vargas Vergara, 2017).

Además, consideramos necesario para seguir avanzando en igualdad, centrar el trabajo coeducativo también en coeducar en nuevas masculinidades más igualitarias, que rompan con la masculinidad hegemónica (Arconada, 2018). Conjuntamente Suberviola (2020), señala que es necesario avanzar hacia una coeducación emocional, teniendo en cuenta la socialización diferencial que existe entre los niños y las niñas, por consiguiente es necesario plantear acciones coeducativas que permitan expresar las emociones con independencia de los roles de género. Necesitamos dibujar una escuela donde los niños y las niñas puedan expresar libremente sus emociones, su identidad de manera igualitaria y superar los desafíos propuestos desde la Agenda 2030 sobre el desarrollo sostenible en materia de igualdad y educación a través de la coeducación.

\section{Referencias}

Alcón Yustas, M. ${ }^{a}$ F. (2005). Acerca del derecho de educación en España de las cortes de Cádiz a la Segunda República. Parlamento y Constitución. Anuario. (9), 33-58. Recuperado de https://cutt.ly/vj6SflB

Arconada, M. A. (2008). Prevenir la violencia de género: El reto de educar alumnos igualitarios. Padres y Maestros, 316, 9-14.

Aristizabal, M. P. (2011). Emakume eta gizonen berdintasuna irakasleen formazioan: Gasteizko Irakasle Eskolaren kasua. Doctoral dissertation, Universidad del País Vasco-Euskal Herriko Unibertsitatea.

Arjona, C.; Díaz, M. \& Rizo, R. (1999). Valores y género en el Proyecto de Centro. Consejería de Educación y Ciencia de la Junta de Andalucía. 
Baena, A. \& Ruiz, P.J. (2009). Tratamiento educativo de la coeducación y la igualdad de sexos en el contexto escolar y en especial en educación física. Aula Abierta, 37(2), 111- 122 .

Ballarín, P. (2001). La educación de las mujeres en la España contemporánea. Síntesis.

Ballarín, P. (2017). ¿Se enseña coeducación en la Universidad? Revista Internacional de Estudios Feministas. 2(1), 7-31.

Bejarano, M. \& Mateos, A. (2014). Género y Sexualidad en la formación inicial de maestros y maestras. ¿Por qué no un currículum sexual? Exedra, Revista científica, número temático, 127-146.

Calatrava, M. A. (2013). Políticas públicas de igualdad en el ámbito de la educación. Balance actual y retos futuros. Profesorado. Revista de Curriculum y Formación del Profesorado, 17(1), 9-27.

Camas, F. (2015). La emergencia de la igualdad de género. Cambios y persistencias de las actitudes de las y los jóvenes en España (1994-2010). [Tesis doctoral inédita Universidad de Granada, Granada].

Colás, P. (2004). La construcción de una pedagogía de género para la igualdad. En, M. A. Rebollo y I Mercado (Coords). Mujer y desarrollo en el siglo XXI: voces para la igualdad (pp 275-291). Madrid: McGraw-Hill.

Conserjería de Educación (2016). II plan estratégico de igualdad de género en educación 2016-2021. Conserjería de Educación y Deporte, Junta de Andalucía. Recuperado de https://cutt.ly/bvqlFfT

Cortes Generales de España. (2006, de 3 de mayo). Ley Orgánica de Educación. Boletín Oficial del Estado. Recuperado de https://cutt.ly/7j6SRn1

Cortes Generales de España. (2013, 9 de diciembre). Ley Orgánica para la mejora de la calidad educativa. Boletín Oficial del Estado. Recuperado de https://cutt.ly/7j6SA97

Cortes Generales de España. (2020, de 25 de noviembre). Proyecto de Ley Orgánica por la que se modifica la Ley Orgánica de Educación. Boletín Oficial de las Cortes Generales, Senado. Recuperado de https://cutt.ly/Uj6SKXH

Cueto, J.L.H., López, J.C.F. \& García, J.A.G. (2016). Reivindicando la memoria. Revista Iberoamericana de Producción Académica y Gestión Educativa, 2(4).

Del Amo, M.C. (2009). La educación de las mujeres en España: de la amiga a la Universidad. CEE Participación Educativa, 11, 8-22. 
Duque, E., Fisas, M. \& Valls, R. (2004). Dialogar y transformar: Pedagogía crítica del siglo XXI. Graó.

Freixas, A., Fuentes-Guerra, M.; Luque, B. y (2007). Formación del profesorado y diferencia sexual. Revista Fuentes, 7.

Gallardo-López, J. A. \& Gallardo-Vázquez, P. (2018). Teorías sobre el juego y su importancia como recurso educativo para el desarrollo integral infantil. Hekademos: revista educativa digital, 24, 41-51.

García-Pérez, R.; Rebollo, Ma A.; Vega, L.; Barragán, R.; Buzón, O., \& Piedra, J. (2014). El Patriarcado no es Transparente: Competencias del Profesorado para Reconocer la Desigualdad. Cultura y Educación, 23(3), 385-397.

Geraldine, M. (1987). La mujer y la instrucción pública de la ley Moyano a la II República. Historia de la educación: Revista interuniversitaria, (6), 193-208. Recuperado de https://cutt.ly/Jj6Du56

Gómez, G. M. N. (2000). Utopías, reformas y contrarreformas en la educación española del siglo XX. Revista de Educación, 37-58. Recuperado de https://cutt.ly/6j6Dds2

González, T. (2010). Aprender a enseñar en el siglo XIX. La formación inicial de las maestras españolas. Revista Electrónica e interuniversitaria de Formación del Profesorado, 13(4), 133-143.

Guattari, F. \& Rolnik, S. (2006). Micropolítica. Cartografías del deseo. Traficantes de Sueños.

Grana, G. I. \& Martín, Z. M. (2015). Las profesoras durante el franquismo: freno a la vanguardia intelectual de las mujeres. Bordón. Revista de pedagogía, 68(3), 59-71. Recuperado de https://cutt.ly/3j6Dkb4

Instituto de la Mujer (2008). Guía de Coeducación: Síntesis sobre la Educación para la Igualdad de Oportunidades entre Mujeres y Hombres. Madrid: Instituto de la Mujer.

Instituto Asturiano de la Mujer (s.f.). Coeducación en la nueva Ley de Educación LOMLOE. Recuperado de https://bit.ly/3ry2Hd9

Jiménez, R.; Vega, L. \& Rebollo-Catalán, A. (2013). Identidad feminista de las profesoras responsables de coeducación y prácticas coeducativas en los centros. Un análisis de las metáforas como reflejo de su formación en género. En Ballesteros-Moscosio, M.A. y Ries, F. (eds.), International Conference Reconceptualizing the Professional Identity of European Teacher (pp. 257 -272). 
Losada, E. (2019). La contribución de las mujeres al boletín de la Institución Libre de Enseñanza. [Tesis doctoral inédita, Universitat de les Illes Balears, Las Islas Baleares].

Montero, A. (2009 21 de mayo). Una ley centenaria: la Ley de Instrucción Pública (Ley Moyano, 1857). Cabás, (1), 1-23. Recuperado de https://cutt.ly/Qj6Dbj1

Organización de las Naciones Unidas. (s. f.). Los Objetivos de Desarrollo Sostenibles. Consultado el 12 de diciembre de 2020. Recuperado de https://cutt.ly/Ej6DTrW

Pérez, G. M. (2000). La enseñanza en la Segunda República. Revista de Educación, núm. Extraordinario, 317-332. Recuperado de https://cutt.ly/Tj6DPXp

Pérez González, A. B., \& Vargas Vergara, M. (2017). "Mamá, tú no sabes". El mantenimiento de la brecha digital por cuestiones de género. Hachetetepé. Revista científica de Educación y Comunicación, (15), 27-35. https://doi.org/10.25267/Hachetetepe.2017.v2.i15.4

Pérez, C., \& Gargallo, B. (2008). Sexismo y estereotipos de género en los textos escolares. Actas XXVI Seminario interuniversitario de teoría de la educación: Lectura y educación, 627- 636.

Pérez Carracedo, L. (12 de enero de 2006). Por la plena integración de los estudios de las mujeres, feministas y de género en el espacio europeo de educación superior. El periódico feminista. Recuperado de https://cutt.ly/Dj6DLmI

Pericacho, F. (2015). La Renovación Pedagógica Española. Un estudio a través de escuelas emblemáticas: de finales del siglo XIX hasta el final de la dictadura franquista (1975). En X, Cid Fernández y M. Carrera Fernández (Coord.), Coloquio de Historia de la Educación, Actas del XVIII Coloquio de Historia de la Educación. Universitat de Vic-Universitat Central de Catalunya.

Quintana, D. (1975). La política educativa de España entre 1850 y 1939. Revista de Educación, no $240,30-40$.

Romero, J. C. (2015). Maestro, ¿cuánto queda para el recreo? Una reflexión antropoeducativa de los lugares escolares. International Journal of Developmental and Educational Psychology INFAD. Revista de Psicología, 1 (1), 323-330.

Sánchez Torrejón, B. (2017). De la coeducación transitando hacia una escuela queer. Actas VI Coloquio "¿Del otro la'o?: Perspectivas sobre sexualidades Queer"

Sánchez Torrejón, B., \& Barea Villalba, Z. (2019). Hacia una escuela violeta: la formación inicial del alumnado de Educación Primaria en coeducación. Tendencias Pedagógicas, 34, 76-92. https://www.doi.org/10.15366/tp2019.34.007 
Sánchez Torrejón., B \& Soto Gutiérrez., A. (2020). Ni princesas ni príncipes la (co)educación, el camino transversal hacia la igualdad en la escuela. En V. Amar (Coord.) Retos para la formación inicial: desde las miradas y las voces de docentes en activo (pp.37-47). Barcelona: Octaedro.

Suberviola Ovejas, I. (2020). La socialización diferencial emocional de género como factor predictor del carácter. IQual. Revista De Género E Igualdad, (3), 80-93.

Subirats, M. (1994). Conquistar la igualdad: la coeducación hoy. Revista Iberoamericana de Educación. 6, 49-78. Recuperado de https://cutt.ly/oj6FFyM

Subirats, M. (2009). La escuela mixta, ¿garantía de coeducación? Revista Participación educativa, 11, 94-97.

Subirats, M. (2010). La coeducación hoy: los objetivos pendientes. Emakunde. Recuperado de https://cutt.ly/7vqxvtK

Subirats, M. (2014). Avances y retos en las políticas y en las prácticas de los géneros. Educar, 50, 85-1000.

Tomé, A. (2017). Estrategias para elaborar proyectos coeducativos en las escuelas. Atlánticas-Revista Internacional de Estudios Feministas, 2(1), 89-116. https://doi.org/10.17979/arief.2017.2.1.1979

Ullastres, Á.M. (2014). Controversias en la educación española. Alianza editorial.

Uria, A. A., Leonet, G. L., \& Morales, M. T. V. (2019). El significado y la evolución del término "coeducación" con el cambio de siglo: el caso de los centros escolares de Vitoria-Gasteiz. Tendencias pedagógicas, (34), 62-75.

Vaillo, M. (2013). Recomendaciones para introducir e innovar en los libros de texto. Instituto de la Mujer del Ministerio de Sanidad, Servicios Sociales e Igualdad. Recuperado de https://cutt.ly/dvqxIck

Vargas, $M^{a}$. E. (2012). El lenguaje sexista en los documentos de planificación de los centros educativos. Espiral. Cuadernos del Profesorado, 5 (9), 37-46. 